\title{
Colorimetric analysis of scanned continuous-tone color images in a Color Managed Digital Photo Printing Workflow (CMDPPW)
}

\begin{abstract}
The purpose of this study is to determine the influence of Color Managed Workflow (CMW) on digital color photo printing. In a CMW, a device profile represents the color characteristics of a device (monitor, scanner, and printer) to be used in the printing workflow. The experiment analyzed the effect of device profiles on scanned and printed continuous-tone color images. The guiding objectives of this study allowed testing of an accepted color management practice to gain a better understanding of the presumptions associated with the application of device profiles. The experiment examined two groups of images [scanned $(K 1)$ and printed $K 2(K=2)$ ]. The conclusions of this study are based upon an analysis of colorimetric data, visual assessment, and associated findings. The data from the experiment revealed that very minor color differences were found between the two groups of photos. The colorimetric data suggests that integration of device profiles is important in a CMW as it allows the process to obtain accurate output colors for a desired purpose.
\end{abstract}

\author{
Haji Naik Dharavath (1) \\ Department of Computer \\ Electronics \& Graphics Technology \\ Central Connecticut State University \\ New Britain, USA
}

Corresponding author: Haji Naik Dharavath e-mail: dharavathh@ccsu.edu

First recieved: 23.09.2019. Accepted: 18.11.2019.

\section{KEY WORDS}

Calibration, color, colorimetry, gamut, profiling, proof

\section{Introduction}

This applied experimental research study is a continuation of a previously published RGB (red, green and blue) to CMYK (cyan, magenta, yellow and black) color managed workflow study (Dharavath, 2010). A continuous-tone color image is composed of a full spectrum of shades and color, from near white to dense black. In a traditional printing (offset, digital offset, gravure or flexography) workflow, the method by which continuous-tone photographic images are transformed to a printable image is called halftoning. In this method, varying percentages of the printed sheet are covered with halftone dots to represent the varying tones in the image. The ink (paste or liquid ink) printed by each dot, of course, has the same density. At normal viewing distance, the dots of a printed image create an optical illusion of a continuous tone image. A digital halftone is a pixel map, with bit depth, that gives the impression of an image containing a range of gray shades or continuous tones. An 8-bit grayscale image contains 256 different levels of gray from white to black. In contrast, a simple digital image could be a binary picture, $[h(x, y)]$, with each point being either completely black or completely white (Pnueli \& Bruckstein, 1996).

Color can be viewed as a science where the optical aspects of color are quantitatively analyzable and measurable. The human eye, however, perceives color more subjectively, which poses a challenge at times for the printing and image reproduction industry. Analyzing the color image by examining its quantitative attributes eliminates the subjective judgment on color quality evaluation of printed colors or colors in nature. Advancements in science and engineering, however, have allowed print and graphic professionals to apply scientific research methods across printing workflow. Applying these methods heightens the importance of proper print production workflow. Workflow is represented through schematic illustrations of activities that reflect the systematic organization of analog and digital devices used during the print and image production process. A print ready 
e-file (.PDF or .JPEG or .PSD or PostScript, etc.) is likely to be manipulated and later printed by an array of output digital devices [computer-to-plate (CTP), digital printers and printing presses]. Given each family of devices tends to create and produce color differently, the challenge is to manage color consistency across the entire workflow (see Figure 1). Analysis of color image quality in the commercial printing workflow requires a Color Management System (CMS) to assist the color producer (printer operator or the designer) in delivering accurate output colors regardless of device color capacities with the use of proper color management techniques (See Figure 1). This method of printing is also applied to digital photo printing.

A majority of the digital images (captured by scanners or digital cameras) for photography purposes are printed by using inkjet printers in the RGB (red, green, and blue) color mode. These printers use more than four process color inks [cyan, magenta, yellow, and black (CMYK)]. This is done by sending the color managed (or color corrected) images via the printer driver supplied by the printer manufacturer. In a commercial printing workflow, inkjet printers can be used as a CMYK printer for final contract proofing purposes. This is done by driving the printer via a raster image processor (RIP) application. The RIP enables the user to integrate the color management system (CMS) by using a set of hardware tools and software applications working together to create accurate color between various input and output devices.
A CMS consists of device profiles (or characterization of devices), which control and document the working performance of the scanner, monitor, and the printer (see Figure 2). A device color transformation engine [Color Management (matching) Module (method) or CMM] is one that interprets the color data among the scanner, display, and the printer. The gamut compensation mechanism of the CMS addresses differences among the color capabilities of the devices in the workflow. A device independent color space [Profile Connection Space (PCS)] is the space through which all color transformation occurs from one device-dependent color space to another. The PCS is based on the color spaces derived from the Commission Internationale de l'Eclairage (CIE) color spaces (CIE color spaces). The color conversion from device dependent color space to device independent color space is achieved by the use of PCS (see Figure 2).

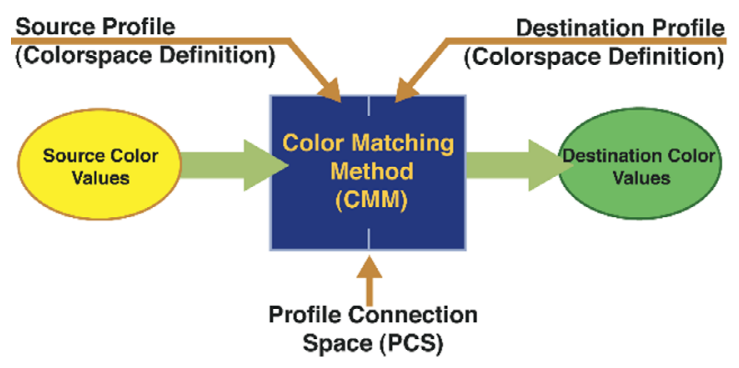

» Figure 2: Schematic of PCS of CMS (Courtesy of Adobe Systems, Inc.)

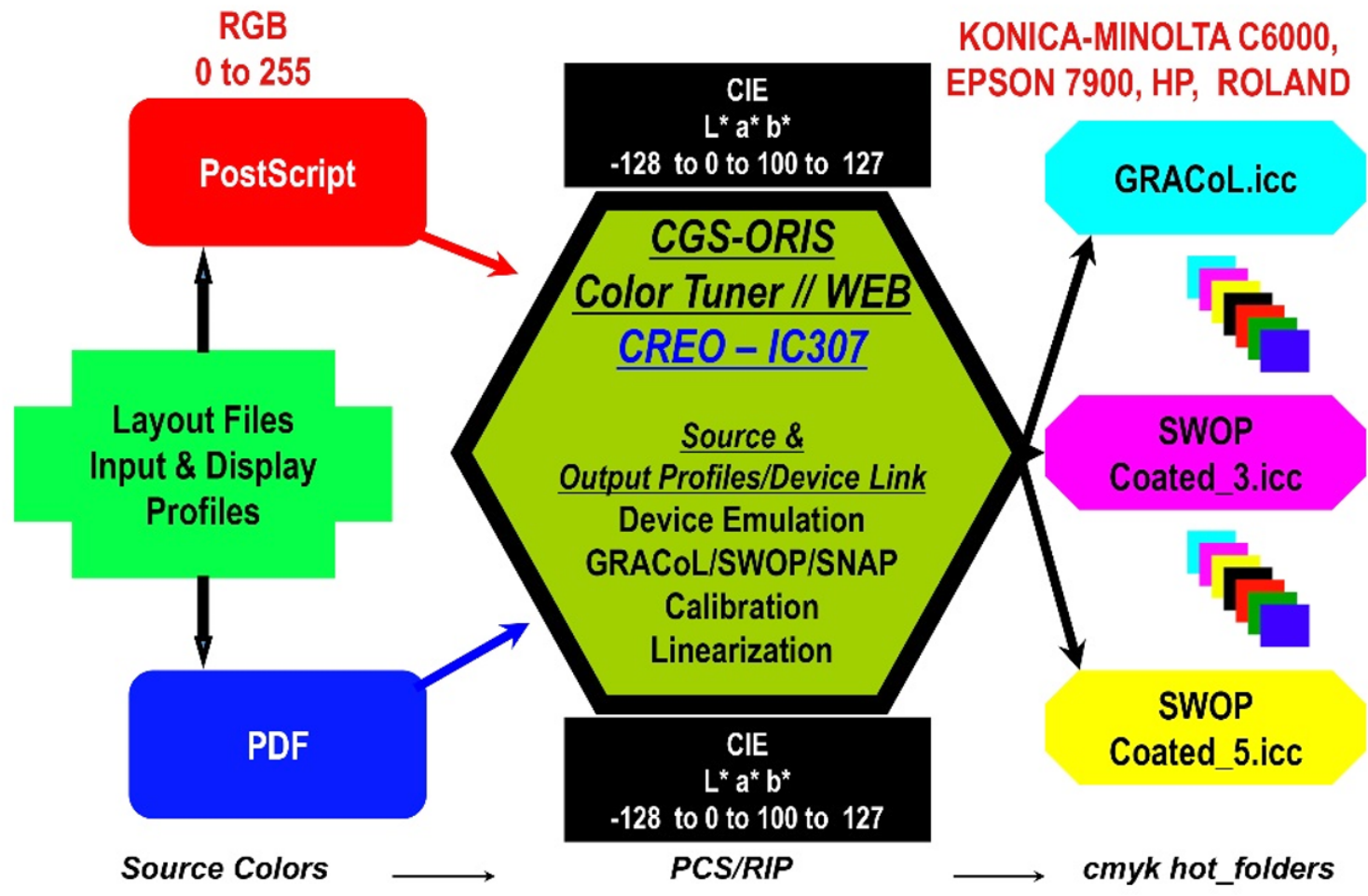

» Figure 1: Schematic Illustration of a Generic Digital Proofing/Printing Workflow 
A device color characterization file (profile) passes in and out of PCS to complete the transformation. The PCS of the CMS is the central hub of the CMS in which a particular color value is considered absolute and not subject to interpretation. Digital print technologies can be described as methods that do not use image carriers such as printing plates or metal imaging cylinders. Inkjet printing utilizes different methods of transferring liquid ink droplets to a substrate to create an image. Inkjet printing technologies today have reached a level of quality that is comparable to traditional printing methods. Laser and inkjet printing generate the majority of digitally printed materials in the industry.

Digital printing technologies today also have reached a level of quality that is comparable to traditional printing methods. The quality of color image reproduction of any type of printing (digital or traditional) is largely influenced by the properties of paper. Paper is considered a commodity but its properties are a long way from being standardized (Wales, 2008). In studies of print quality using process color ink systems, there are a number of variables, such as dot gain, that may cause tonal variations, and can have negative influences on the accuracy of color reproduction. Measuring, recording/ documenting, and analyzing certain color photo print attributes/variables (value/lightness, hue, and chroma) may enable the technologist to make controlled adjustments and then check these variables to see if positive changes can be affected and maintained.

\section{Purpose of the research}

The experiment was conducted in a color managed digital photo printing workflow (CMDPPW). The purpose of this experimental study was to determine the differences that exist among the printed colors hue $(\Delta \mathrm{H})$ deviation [Cyan, Magenta, Yellow, and Black (CMYK) + Red, Green, and Blue (RGB)], and the overall deviation $(\triangle E)$ of printed colors $(C M Y K+R G B)$. Another reason for the study was to determine the chroma deviation $(\triangle C)$ of the two printed color images, namely: Scanned Image and Printed Image (groups, $K=2$ ). This was accomplished by comparing the printed image colorimetric values against the reference colorimetric values of the scanned color continuous-tone image. The following guiding objectives were established to complete the study:

1. Determine the deviation in the color hue $(\triangle H)$ of $C M Y K+R G B$ colors between the scanned photo and the printed photo.

2. Determine the deviation in the average/ overall color $(\triangle E)$ of $C M Y K+R G B$ between the scanned photo and the printed photo.

3. Determine the deviation in the printed colors $(\triangle C)$ chroma of CMYK+RGB between the scanned photo and the printed photo.

\section{Limitations of the Research}

For this experiment, there were limitations to the technology used within the graphics technology program laboratory. Prior to scanning and printing and measuring the samples, the digital color devices (scanner, monitor, and printer) and color measuring device (spectrophotometer) used in the workflow were calibrated against the recommended reference. Also, the input (scanner) and output (printer) devices were characterized (profiled) by using the ICC/ISO/ANSI compliance software, test targets, and color measuring instruments (see Table 1), such as: X-Rite i1PROFILER 1.80, IT8.7/2 Reflective Scanner Target, Custom RGB printer target with 1000 patches, and X-Rite Eye-One PRO Spectrophotometer with i1iO Scanning Table. The print condition associated with this experiment was characterized by, but not restricted to, inherent limitations: colored images (continuous-tone color images and Kodak color targets) chosen for printing, desired rendering intent applied, type of digital printer for proofing/printing, type of paper for printing, type of toner, resolution, and use of customized color input and output profiles, calibration data applied, and so on. Several variables affected the facsimile reproduction of color images in the CMDPPW, and most of them were mutually dependent. The scope of the research was limited to the inkjet digital printing system (printing proof/printing) and other raw materials and color management and control applications (data collection, data analysis, profile creation, and profile inspection, etc.) used at the university graphics laboratory. Findings were not expected to be generalizable to other CMDPPW environments. It is quite likely, however, that professionals could find the method used and the data of this article meaningful and useful. The research methodology, experimental design, and statistical analysis were selected to align with the purpose of the research, taking into account the aforementioned limitations.

\section{Research methodology}

The methodology for this experiment was adopted from a previously published RGB to CMYK color managed workflow study (Dharavath, 2010). The experiment was conducted in a RGB color managed digital photo printing workflow (CMDPPW) to determine the differences that exist in the color quality of scanned images vs. printed images, based on the colorimetric evaluations. Each type of image [source (scanned) and printed] used in the experiment was considered as a group, noted by letter " $K$ " $(K=2)$. This study was focused on the measurement of color prints, printed on 55 LB Instant-Dry Gloss Photo Paper [200 grams per square $\left(200 \mathrm{~g} / \mathrm{m}^{2}\right)$ ] using an inkjet printer. The digital color printer used in this experiment was an HP Designjet Z3200 Photo Inkjet printer. Scanned Images were color managed by using device profiles (scanner and printer) in an Adobe 
Photoshop-CC application, and saved in a tagged image file format (.TIFF). Saved images were placed into a one-page Adobe InDesign-CC layout of 22" W x 17" H size and a portable document format (.PDF) file was created without any compression technique and used for a photo printing purpose in the experiment. Detailed explanations of scanning continuous-tone images, scanner and profiling processes, and applying profiles to the scanned images are presented in the following sections.

\section{High-End Flatbed Scanner}

The scanner used in this experiment was an Epson Expression 11000XL Graphics Arts scanner (11" H x 17 " W). It is capable of scanning images with a maximum of 2400 pixels per inch (ppi) resolution, with a 3.80 maximum density (Dmax) and 24 to 48-bit color ( 8 bit per color channel or 16 bit per color channel). It utilizes Epson proprietary Color Matrix-CCD (charged coupled device) technology. The front-end of the scanner is powered by SilverFast Scanning Software [Version 8.0 (2018)] of Laser Soft Imaging, Inc.

Prior to scanning the photos for the experiment, the scanner was calibrated by using the vendor provided color target (Laser Soft Imaging, IT8.7/2-1993 Reflective Target with 288 color patches). The outcome of the calibration was $\Delta E=0.90$. Upon the completion of the calibration process, a 24 step grayscale (as part of Kodak IT8.7/2 target image, see Figure 5) was scanned in the RGB mode for 8 bits per color channel with 200 ppi to test the linearity of the scanner response to (see Figure 3) given input. Prior to printing the grayscale image, it was opened in the Adobe Photoshop-CC application and the gradations per each color channel were recorded and averaged for later use in the experiment. The scanned grayscale was printed on the HP Designjet Z3200 Inkjet printer and the densities of 24 steps were measured by using an X-Rite EyeOne Spectrophotometer. Measured densities and the gradations were used to create the linearization chart of the scanner (See Figure 3).

An ANSI/ISO (American National Standard Institute/ International Standards Organization) Kodak IT8.7/2 scanner target was scanned at 200 pixels per inch to create the profile for the EPSON Expression 11000XL High-end flatbed scanner that was used in the experiment. Prior to scanning the target, all the color management and color correction options were disabled in the scanning software. The scanner profiling is the process of determining the precise color characteristics of a scanner. To build the scanner profile with the use of IT8.7/2 target, the scanned target was cropped and run through X-Rite i1Profiler software [Version 1.8.0 (2018)]. During the

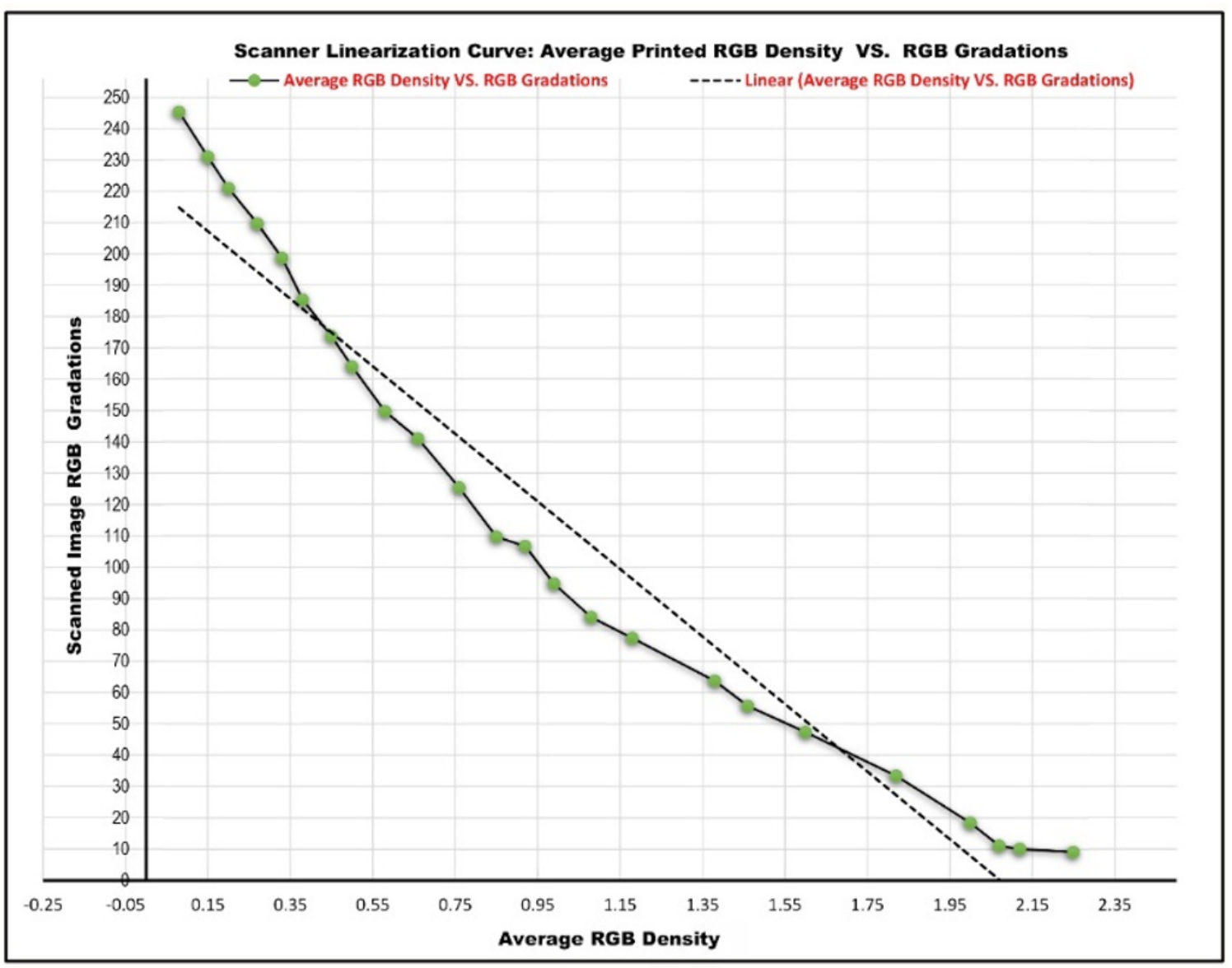

» Figure 3: Scanner Linearization Curve 
profiling process, the software compares the color data generated by the scanner to TDF (Target Definition File) or the known colorimetric values of the pre-measured target (IT8.7/2 Target Q-60 reference data file) to generate the profile. Upon creating the profile it was saved at the right location on the computer (C: $\backslash$ Windows \System32\spool(drivers(color) to make it function properly via Adobe-Photoshop-CC (see Figure 4). Device profiles which were not saved in the right location of the computer will not be accessible to use in the Adobe-Photoshop-CC application for color management purposes.

\section{Continuous-tone (contone) Images for Scanning}

The test image (see Figure 5) contained the following scanned images: three generic images for subjective/ visual assessment/evaluation of color, an ISO IT8.7/2 Kodak Scanner Target and a Kodak Color Control Target. All these images are classified as color continuous-tone RGB color images. These images were acquired for teaching purposes almost ten years ago, used repeatedly and were well preserved. These images present an acceptable range of densities and tonal values for facsimile reproduction of color. After completing the scanner calibration, characterization, and linearization, all five contone images presented on the test target (see Figure 5) were scanned at $150 \%$ scaling ratio (1:1.5) with 450 ppi resolution in 8 bit per color channel mode (RGB) for further reproduction, which includes the Kodak IT8.7/2 Reflective color target and the Kodak color control target.
At the scanning stage, no color management or color correction techniques were applied to the contone images. During the scanning, the scanner transforms the color information of the contone images into digital data from analog signals. The scanned images were saved in the .TIFF format without any compression for later use in the experiment. The reflective (scanned or printed) IT8.7/2 target consists of 264 color patches (including CMYK+RGB and grayscale) which can be measured by using the spectrophotometer or densitometer. Of the 264 patches, 24 patches were the part of grayscale. Prior to scanning IT8.7/2 target, the CIE L* $\mathrm{a}^{*} \mathrm{~b}^{*}$ values were measured on the color patches (CMYK+RGB) at highlight, mid-tones, shadow, and solid colors. Data associated with the established objectives of this study were extracted from this target [Kodak IT8.7/2 Color Reflective (scanned and printed)] only (see Figure 5).

\section{Digital Photo Inkjet Printer}

An HP Designjet Z3200 Photo RGB printer was used in the experiment. An RGB printer target with 1000 patches (see Figure 6) was created in .TIFF format with 100 ppi resolution by using an X-Rite i1PROFILER application to suit the patch measurements on the i1iO-i1Pro 2 Spectrophotometer. The target image was placed into an Adobe InDesign-CC layout of 12 " W $\times 18^{\prime \prime} \mathrm{H}$ size and a .PDF file was created without any image/color compression technique.

Prior to printing the patches/target image, the printer was calibrated according to its manufacturer specifications.

$L^{*}$

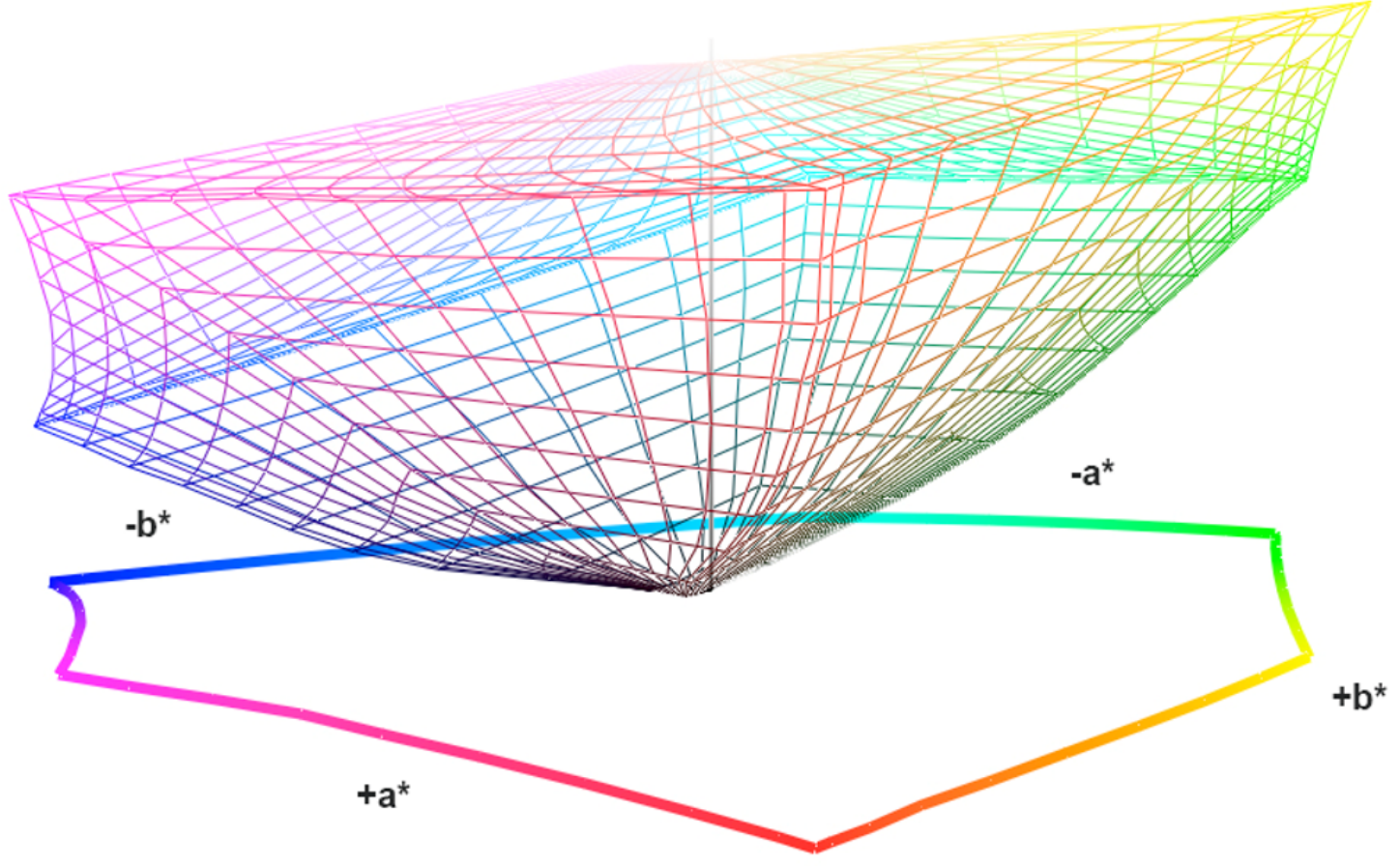

» Figure 4: $3 D L^{*} a^{*} b^{*}$ Gamut of the EPSON Expression 11000XL Scanner Profile 
Colorimetric Analysis of Scanned Continuous-tone color Images in a Color Managed Digital Photo Printing Workflow (CMDPPW) Test Target for Digital Proof/Print Production Workflow Optimization


Printed on HP Designjet 23200 Photo Printer
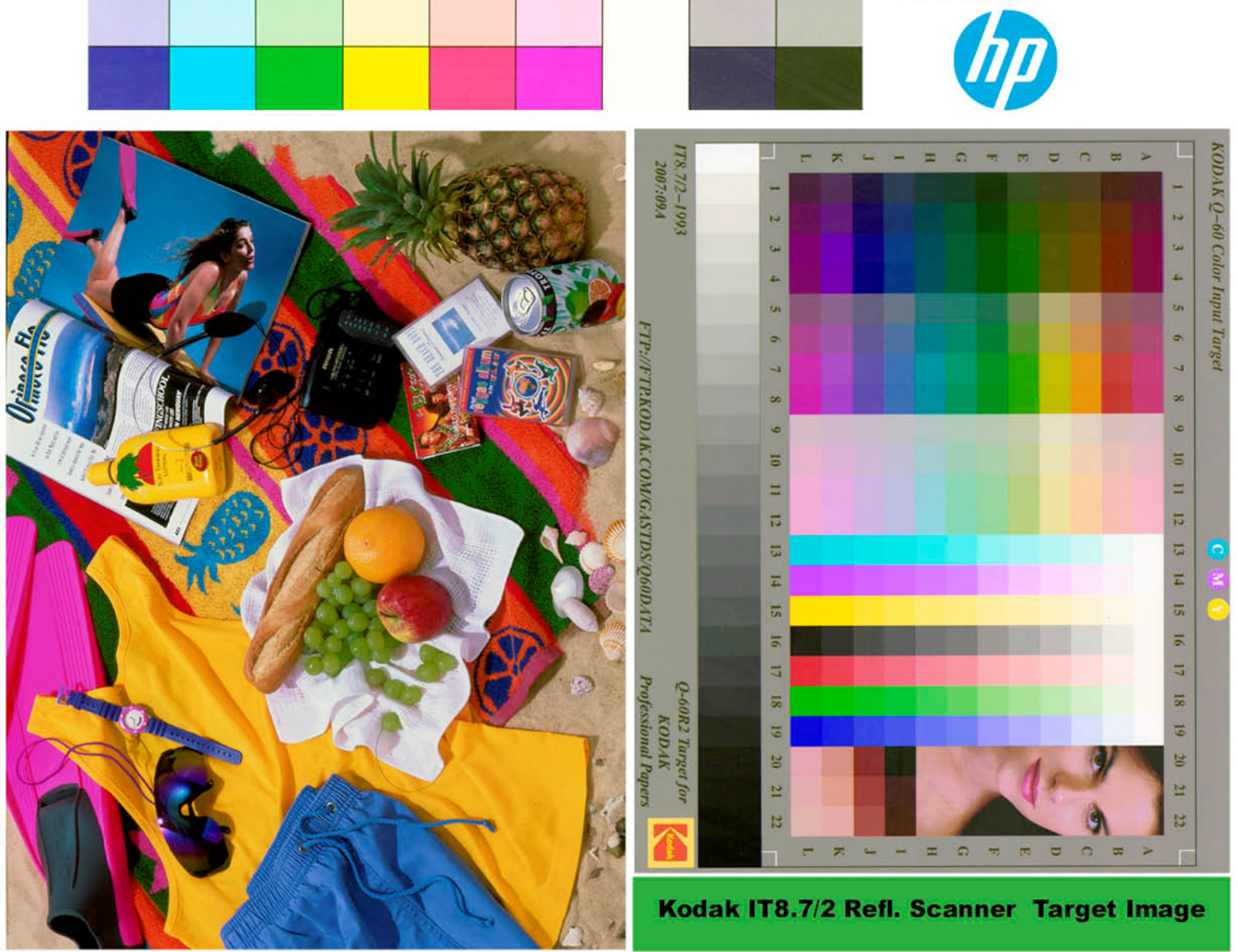

Photos (ALL IMAGES IN THIS LAYOUT) Scanned on EPSON Expression 11000XL Graphic Arts Scanner at 450 PPI with SilverFast (LaserSoft Imaging) Scanning Software, V.8.0 [2018]

» Figure 5: Test Image for Printed Colors Evaluation 


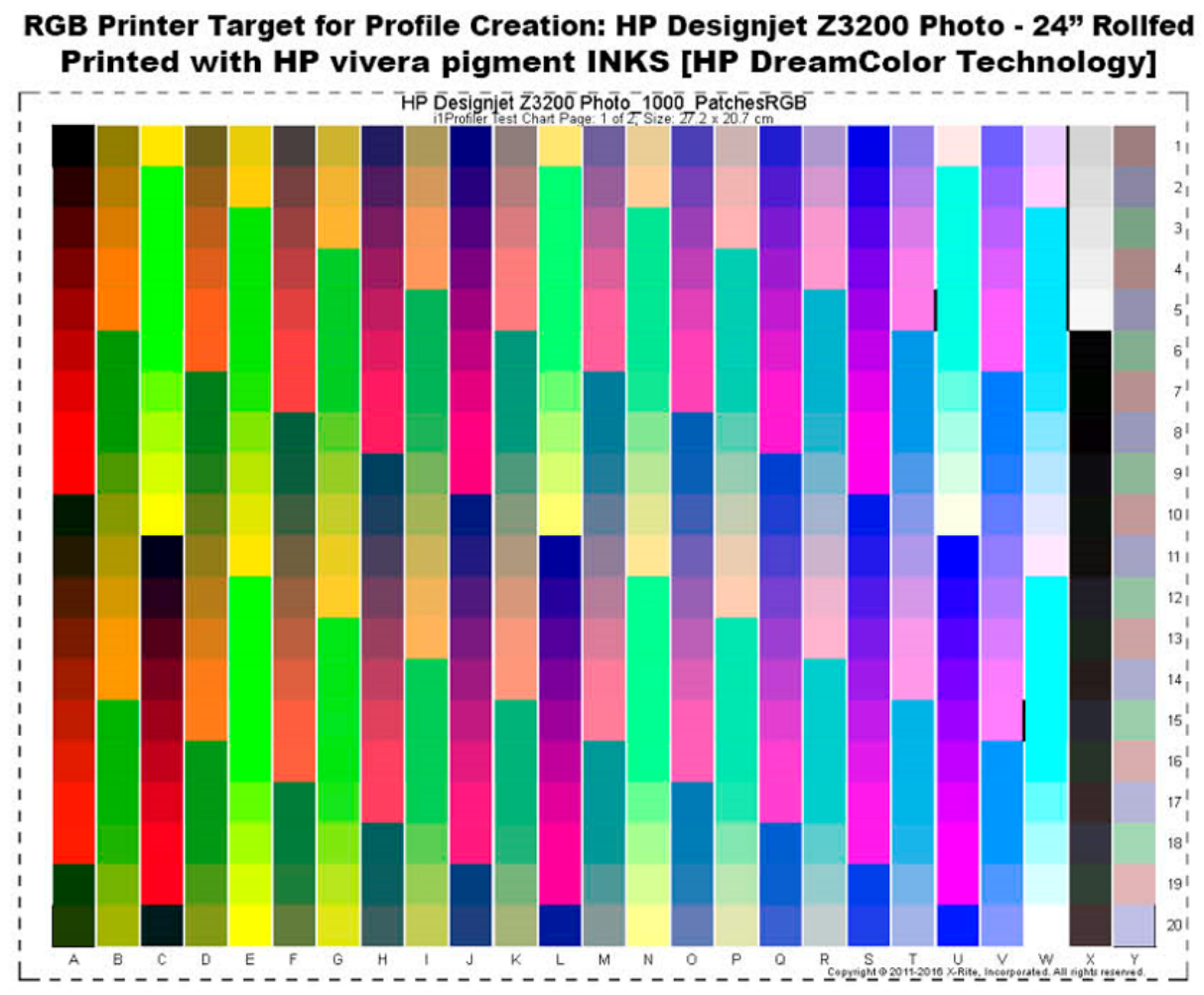

2 Pages, each 8.5" $\times 11$ " dimension Custom Target Image in RGB Mode with 100ppi resolution 1000 Patches, each patch is 0.375 " $\times 0.375$ " in dimension For Single Scan Measurement for MO mode

on an X-Rite i1iO Table with i1Pro 2 Spectrophotometer via X-Rite i1PROFILER 1.8.0



» Figure 6: RGB Printer Target for Printer Profile 
A calibration process means standardizing the performance of the devices according to the device manufacturer specifications so that the results of the devices are repeatable. No raster image processor (RIP) application was used to print the target. The target was printed via the printer driver from the .PDF file. No color management or color correction techniques were applied during the printing. Printed patches were measured in $\mathrm{CIE} \mathrm{L}^{*} \mathrm{a}^{*}$ $b^{*}$ space using the i1PROFILER application with an X-Rite spectrophotometer and the data was run through this application. The printer profile was created and stored at the right location on the computer (C: $\backslash$ Windows \System32\spool\drivers\color). The profile format version is 4.00. Upon completing all the device profiles used in the experiment, they were inspected with ColorThink Pro profile inspection software [Version 3.0.5 (2019)] for profile accuracy, extracting $L^{*} a^{*} b^{*}$ values and creating profiles $L^{*} a^{*} b^{*}$ graphs (see Figure 7). Visual examination of the device profiles indicate that the printer profile is smaller than the scanner profile (see Figure 7). Each profile is an indication that they have different color capabilities because they represent different imaging devices.

\section{Applying Device Profiles to Color Manage Scanned Images for Printing}

All the scanned photos were color managed in the Adobe Photoshop-CC application by embedding the device profiles (scanner and printer) with a relative colorimetric rendering intent (see Figure 7). As described earlier, a test image for color evaluation was created for the digital photo printing for the experiment (see Figure 5). The test image (the target) contained the following scanned continuous-tone color managed images: Kodak IT8.7/2 reflective color target with 264 patches for colorimetric data collection, Kodak color control target with 18 patches for colorimetric data collection, and three scanned photos for subjective/visual assessment. Each scanned photo was opened in the Adobe Photoshop-CC, a scanner profile was assigned to the photo (RGB) and converted to destination colors ( $R G B$ to RGB) with the printer profile. The same steps were repeated for all the scanned photos and saved in .TIFF format for layout and printing purpose. No other color correction or color manipulation techniques were applied to these images. In Adobe Photoshop-CC, CIE $L^{*} a^{*} b^{*}$ values were extracted from the color managed Kodak IT8.7/2 scanned photo. The $L^{*} a^{*} b^{*}$ values were collected at the solids, shadows, midtones, and highlight areas of the CMYK+RGB colors for comparison with the printed photo and the image was then saved. Later, the saved color managed scanned photos were imported into a one page layout of $22^{\prime \prime} \mathrm{W} \times 17^{\prime \prime} \mathrm{H}$ size (Adobe InDesign-CC) and a .PDF file was created.

No RIP application was used to print the .PDF layout (color managed .PDF file). A total of 5 proofs were printed on the HP Designjet Z3200 Photo (RGB) Printer. Printing was done from the .PDF file via the printer driver with an application managed color option. This option was available in this printer driver interface (printing interface). This print method preserves the colors of embedded device profiles in the color managed scanned photos. Upon printing, the CIE L* a* $b *$ values of all the printed proofs were measured with an X-Rite Eye-One PRO spectrophotometer using MeasureTool 5.10 (X-Rite ProfileMaker 5.10) application interface. The $L^{*} a^{*} b^{*}$ values were collected at the printed (destina-

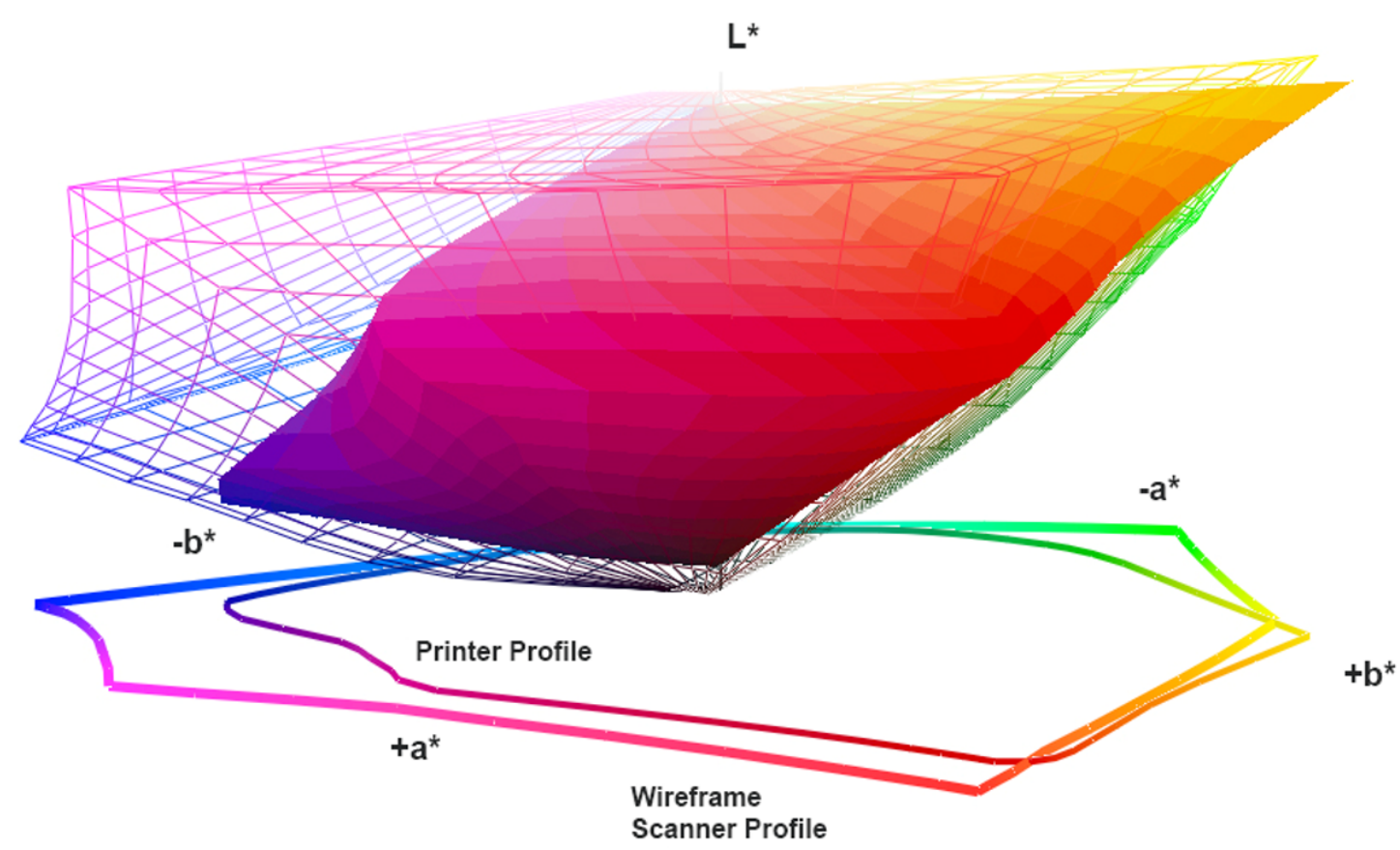

» Figure 7: $3 D L^{*} a^{*} b^{*}$ Gamut Comparison of Device Profiles (RGB VS. RGB) 
tion colors) solids, shadows, midtones, and highlight areas of the $\mathrm{CMYK}+\mathrm{RGB}$ colors for the colorimetric comparison with the scanned image (source color) values. Further color quantification of this research is presented in the DATA ANALYSIS section. Table 1 presents the variables, materials, conditions, and equipment associated with this experiment (see Table 1).

\section{Data analysis}

A total of five printed proofs were analyzed for objective evaluation. The data from the proofs were collected by using an X-Rite Eye-One Spectrophotometer with a MeasureTool 5.10.0 interface. Only the CIE $L^{*} a^{*} b^{*}$ values of $C M Y K+R G B$ colors were measured at the solid, shadows, midtones, and highlight color areas on the printed proof. Collected data from the proofs were averaged (average $L^{*} a^{*} b^{*}$ ). Further colorimetric computations were applied to see the noticeable col- or differences that exist between the scanned photos vs. the printed photos. Colorimetric equations presented in the literature review section were used to manually compute the $\mathrm{c}^{*}$ and $\mathrm{h}^{*}$ of each color. Also, the $\Delta \mathrm{E}, \Delta \mathrm{C}$, and $\Delta \mathrm{H}$ were calculated to determine the color (CMYK+RGB) deviations/variations between the two groups of colored photos/images $(K=2)$.

Subjective judgment on color difference was not used (or applied) in this study. The subjective judgment of color difference could differ from person to person. For example, we see colors in an image not by isolating one or two colors at a time (Goodhard \& Wilhelm, 2003), but by mentally processing contextual relationships between colors where the changes in lightness (value), hue, and chroma (saturation) contribute independently to the visual detection of spatial patterns in the image (Goodhard \& Wilhelm, 2003). In comparing the color differences between two colors, a higher deviation $(\Delta \mathrm{E} / \Delta \mathrm{H} / \Delta \mathrm{C})$ is an indication that there is more difference

\section{Table 1}

Experimental and Controlled Variables

\begin{tabular}{|c|c|}
\hline Variable & Material/Condition/Equipment \\
\hline Test Image & Custom, $22^{\prime \prime} \mathrm{W} \times 17^{\prime \prime} \mathrm{H}$ with 5 Contone Images \\
\hline Scanner & EPSON Expression 11000XL High-end Scanner \\
\hline Scanned Photos resolution & 450 ppi \\
\hline Scaling Ratio & $150 \%(1: 1.5)$ \\
\hline Scanner Target & ANSI/ISO Kodak IT8.7/2 Reflective \\
\hline Profiling Software & i1PROFILER 1.8 .0 by X-Rite \\
\hline ICC Profiles Version & 4.0 \\
\hline Profile Inspection Software & ColorThink Pro 3.0 .5 by Chromix \\
\hline Image Editing Software & Adobe Photoshop-CC \\
\hline Page Layout Software & Adobe InDesign-CC \\
\hline Color Management Module (CMM) & Adobe (ACE) CMM \\
\hline Rendering Intent & Relative Colorimetric \\
\hline Computer \& Monitor & Dell OPTIPLEX 9020 \\
\hline Raster Image Processor (RIP) & None \\
\hline Printer & HP Designjet Z3200 Inkjet Photo (RGB) \\
\hline Printer Driver & HP Designjet Z3200 \\
\hline Printer Target & Custom RGB (1000Patches) \\
\hline Target Resolution & 100 ppi \\
\hline Inks & HP vivera pigment INKS \\
\hline Number of Inks & $\begin{array}{l}12 \text { (Gloss Enhancer, Gray, Blue, Green, Magenta, Yellow, Light Magenta, } \\
\text { Light Cyan, Photo Black, Light Gray, Matte Black, and Red) }\end{array}$ \\
\hline Paper (Rollfed) & HP Universal Instant-dry Gloss Paper \\
\hline Type of Illumination/Viewing Condition & D50 \\
\hline Color Measurement Device(s) & X-Rite Eye-One PRO Spectrophotometer with i1iO Scanning Table \\
\hline Measurement Condition & MO, UV included \\
\hline Data Collection/Analysis Software & X-Rite Measure Tool \& MS-Excel \\
\hline File Formats & .TIFF, .PDF, and .ICC \\
\hline
\end{tabular}


and a lesser deviation is an indication of less difference. Analyzed results are presented in the following sections.

\section{Overall Color Variation $(\Delta \mathrm{E})$ in the Solid Color area of Scanned Photo vs. Printed Photo}

The CIE L* $a^{*} b^{*}$ values associated with the CMYK+RGB colors in the solid color area of Scanned Photo vs. Printed Photo are compiled in Table 2. Numerical color differences $(\Delta E)$ were found when comparing the color in the solid area of the scanned image and in the printed image at all seven colors (CMYK+RGB). Also, noticeable visual color differences were found in the solid color area [lightness, color hue and chroma]. Overall, both groups of images have similar colors in the solid area (see Figures 8 to $9 \mathrm{~B})$, with the exception of the printed proof consisting of higher color values for red, magenta, and blue.

This higher color deviation (red, magenta, and blue) could be the result of the substrate (paper) and inks used (age, condition, quality, etc.). These are the darker colors which produced lower $L^{*}$ value and in turn affected the higher deviation. The $2 \mathrm{D}$ color gamut comparison (see Figure 8) reveals that the color of the scanned photo closely matches the printed photo. The goal was to determine the deviations among various attributes of color between these two groups of photos [scanned (K1) vs. printed (K1)]. The compar- ison is an indication that, in a color managed workflow (CMW), color matching of a target image can be achieved from device to device regardless of device color characterization and original colors. Subjective judgment was not used for the color comparison.

\section{Table 2}

Overall Color Variation in the Solid area of CMYK+RGB: Scanned Photo vs. Printed Photo

\begin{tabular}{|c|c|c|c|c|c|c|c|}
\hline & \multicolumn{3}{|c|}{$\begin{array}{l}\text { K1 (Scanned } \\
\text { Photo) }\end{array}$} & \multicolumn{3}{|c|}{$\begin{array}{l}\text { K2 (Printed } \\
\text { Photo) }\end{array}$} & \multirow{2}{*}{\begin{tabular}{|l} 
Color \\
Difference \\
\end{tabular}} \\
\hline & $\mathrm{L}^{*}$ & a* & b* & L* & $\mathrm{a}^{*}$ & b* & \\
\hline Color(s) & \multicolumn{3}{|c|}{ Color 1} & \multicolumn{3}{|c|}{ Color 2} & $\Delta \mathrm{E}$ \\
\hline Yellow & 78 & 11 & 83 & 78 & 12.3 & 82.4 & 1.43 \\
\hline Red & 40 & 54 & 51 & 41.2 & 55.8 & 47.6 & 4.03 \\
\hline Magenta & 46 & 60 & -39 & 44.8 & 62.3 & -41.4 & 3.53 \\
\hline Blue & 23 & 31 & -65 & 21.2 & 34.7 & -69.1 & 5.81 \\
\hline Cyan & 54 & -36 & -41 & 55.4 & -35 & -40.5 & 1.79 \\
\hline Green & 45 & -46 & 36 & 45.1 & -47.2 & 35.9 & 1.21 \\
\hline Black & 17 & 1 & 0.0 & 14.1 & 0.2 & 0.4 & 3.03 \\
\hline
\end{tabular}

Chroma and Hue Variation $\left(\Delta C^{*} \& \Delta H^{*}\right)$ values of the Solid Color area of a Scanned Photo vs. a Printed Photo are presented in Table 3. Interpreting $L^{*} c^{*}$ *h color space data is similar to $L^{*} a * b *$, but the data describes color differently using cylindrical coordinates instead of rectangular coordinates (see Figures 8 to $9 B$ ). In this color space, L* indicates lightness,

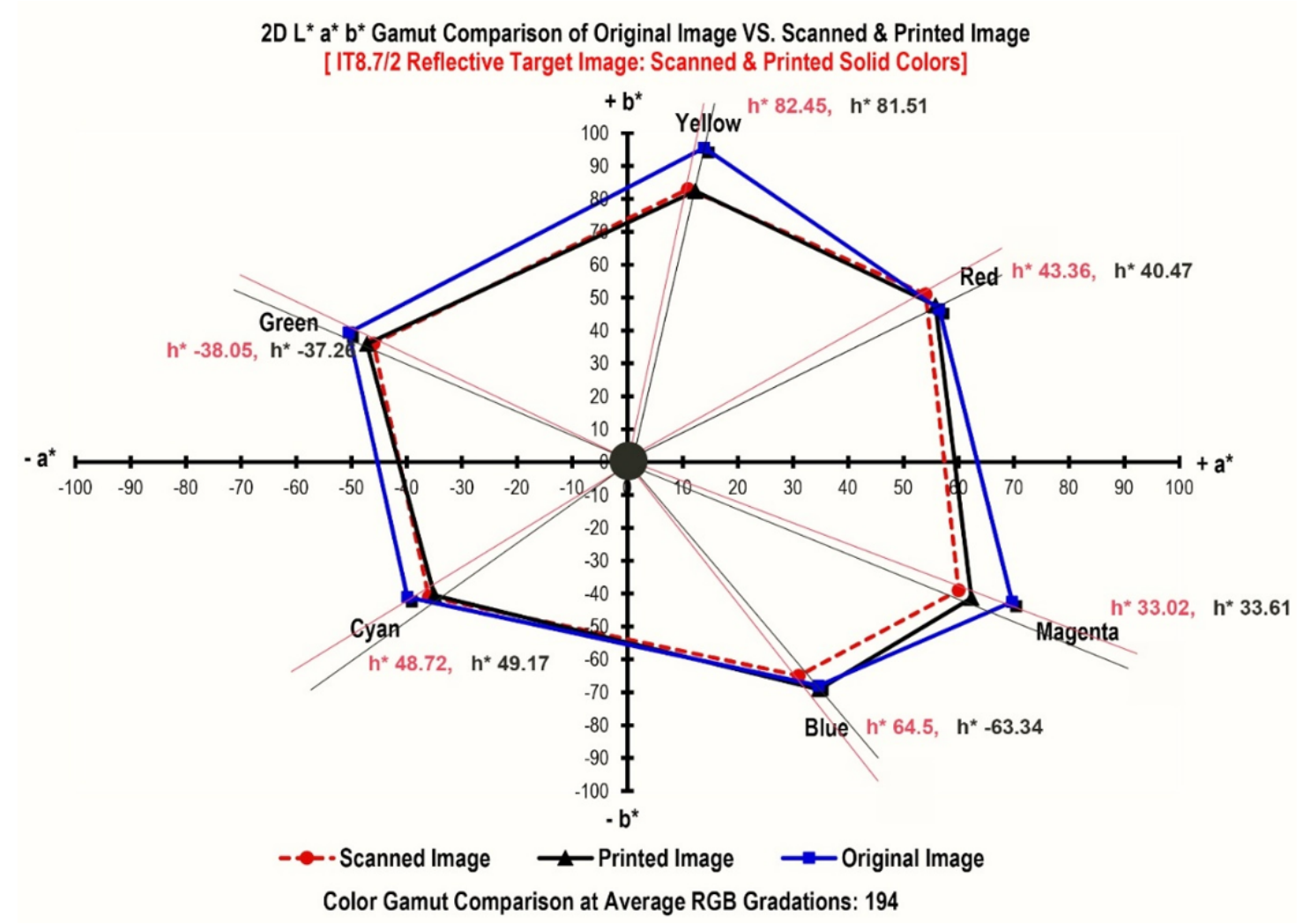

» Figure 8: CIE L* $a^{*} b^{*}$ Model (2D) for Solid Colors Comparison of Scanned Photos and Printed Photo vs. Original Photo 
$C^{*}$ represents chroma, and $h^{*}$ is the hue angle of the color. Deltas (deviations) for lightness $\left(\Delta \mathrm{L}^{*}\right)$, chroma $\left(\Delta C^{*}\right)$, and hue $\left(\Delta H^{*}\right)$ may be positive $(+)$ or negative $(-)$. The cyan, green, and red color are at $-\Delta L^{*}$. In comparison with the printed colors, these scanned colors are darker in lightness (see Figures 8 to 9B).

The $+\Delta L^{*}$ values indicate that the remaining colors are lighter. The $-\Delta C^{*}$ of blue, green, and magenta colors indicate that they are duller in comparison with the other of the same colors in the reproduction. The $+\Delta C^{*}$ of cyan, yellow, red, and black colors are brighter. The positive $\Delta H^{*}$ values indicate that these colors fall in counterclockwise (or clockwise) to one another, meaning these colors do not match $100 \%$.

\section{Table 3}

Chroma and Hue Variation in the Solid area of CMYK+RGB: Scanned Photo vs. Printed Photo

\begin{tabular}{l|l|l|l|l|l}
\hline & \multicolumn{2}{|l|}{ K1 (Scanned Photo) } & & \multicolumn{2}{l}{ K2 (Printed Photo) } \\
\hline & \multicolumn{2}{|l|}{ c* $^{*}$} & h* & & \multicolumn{2}{l}{ c* } & \multicolumn{1}{l}{ h* } \\
\hline Color(s) & Color 1 & & \multicolumn{2}{l}{ Color 2 } \\
\hline Yellow & 83.73 & 82.45 & & 83.31 & 81.51 \\
\hline Red & 74.28 & 43.36 & & 73.34 & 40.47 \\
\hline Magenta & 71.56 & -33.02 & & 74.80 & -33.61 \\
\hline Blue & 72.01 & -64.50 & & 77.32 & -63.34 \\
\hline Cyan & 54.56 & 48.72 & & 53.53 & 49.17 \\
\hline Green & 58.41 & -38.05 & & 59.30 & -37.26 \\
\hline Black & 1.00 & 0.00 & & 0.45 & 3.43 \\
\hline
\end{tabular}

» Figure 9: Color Variations $\left(\Delta L, \Delta C^{*}, \Delta H\right.$, and $\left.\Delta E\right)$ of Scanned vs. Printed [Colors presented in this chart do not represent actual K1 vs. K2 colors]

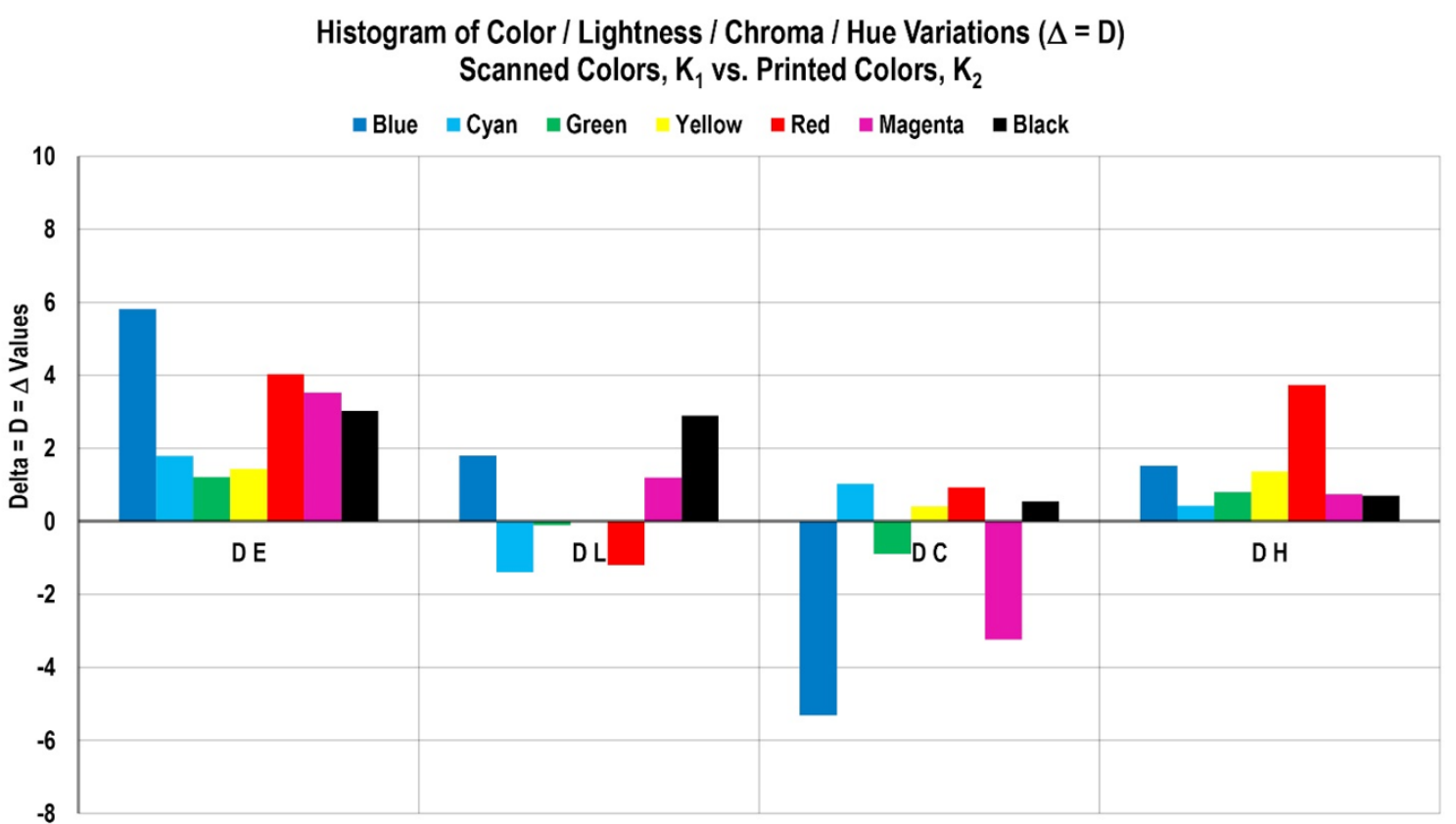

» Figure 9A: Histogram of Color Variations ( $\Delta L, \Delta C^{*}, \Delta H$, and $\Delta E$ ) of Scanned vs. Printed [Colors presented in this chart do not represent actual K1 vs. K2 colors] 


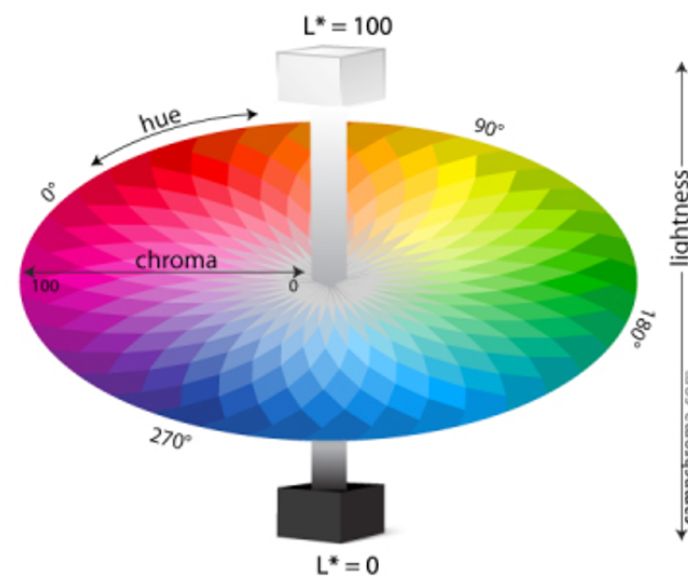

» Figure 9B: CIE 3D L* $C^{*} h^{*}$ Color Coordinates

\section{Conclusions}

The contents of this research report demonstrated the complete color managed digital photo printing workflow (CMDPPW) for RGB to RGB conversion. The conclusions of this study are based upon an analysis of the data and major findings. The findings of this study represent specific printing or testing conditions. The contone photos, scanner, printer, instrument, software, and paper that were used are important factors to consider when evaluating the results. The findings of the study cannot be generalized to other CMDPPW. However, other graphic arts educators, industry professionals, and researchers may find this study meaningful and useful. For example, educators can implement a similar or presented model (or method) to teach color management for various input and output devices.

The findings of this research show that scanned photo colors vs. printed photo colors are very similar (image scanned vs. printed is identical). This is due to the integration of color management in an existing workflow. As seen in the device profiles (see Figure 9), all the devices present a totally different color gamut. However, applying the device profiles to scanned photos offered more flexibility and control over colors and tonal values in reproducing color photos. This process enables us to accurately and consistently reproduce color with predictable results from device to device. Application of device profiles to scanned photos cannot match colors with the original photo. It is impossible to accomplish this. Also, it may not be possible to match exactly a color gamut of one device to another device. The goal of applying device profiles to scanned images is to ensure that colors captured are a close match to that of the output of the printer.

As a result of this experiment, it is evident that color management works and it offers more flexibility and control over color reproduction images. Also learned was that only the optical aspects of color are quantitatively analyzable and measurable because humans perceive color subjectively. It will be hard to document and measure the color values we see or detect. Additionally, the implementation of CMDPPW is costly, time consuming and a tedious process. It does, however, benefit those who implement this workflow to get consistent color from device to device. Future study is needed to determine the color image differences of color management workflow vs. non-color management workflow. This study was limited to an inkjet printer only. Future studies can be conducted by using other printing processes.

\section{References}

Dharavath, H. (2010) Integration of a Color Management System (CMS) within the Graphic Communications Laboratory's Digital Proof/Print Production Workflow. Visual Communications Journal. 46

(1), 27-34. Available from: http://gceaonline.org/ wp-content/uploads/2014/08/VCJ_Spring_2010low4. pdf\#page=28 [Accessed: 10th July 2019].

Goodhard, M. M., \& Wilhelm, H. (2003) A new test method based on CIELAB colorimetry for evaluating the permanence of pictorial images. Grinnell, Wilhelm Imaging Research, Inc. Available from: https://pdfs.semanticscholar.org/7c3e/5df29ee8e0bc11208cf8dfb902c2e45024c0. pdf?_ga=2.165255487.1855428551.1574088224155791326.1552396228 [Accessed: 20th August 2019]. International Organization for Standardization. (2017) ISO 13655:2017. Graphic technology Spectral measurement and colorimetric computation for graphic arts images. Geneva, International Organization for Standardization.

Pnueli, Y. \& Bruckstein, A. (1996) Gridless Halftoning: A Reincarnation of the Old Method. Journal of Graphical Models and Image Processing. 58 (1), 38-64. Available from: doi: 10.1006/ gmip.1996.0003 [Accessed: 18th August 2019]. Wales, T. (2008) Paper: The Fifth Color- IPA Bulletin. Alexandria, IDEAlliance/International Prepress Association (IPA). Available from: http://idealliance.org/ files/2008_03tech.pdf [Accessed: 20th August 2019]. 


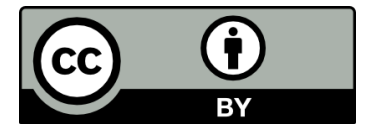

(C) 2019 Authors. Published by the University of Novi Sad, Faculty of Technical Sciences, Department of Graphic Engineering and Design. This article is an open access article distributed under the terms and conditions of the Creative Commons Attribution license 3.0 Serbia (http://creativecommons.org/licenses/by/3.0/rs/). 